\title{
EDITORIAL
}

\section{Defects of immune surveillance offer new insights into the pathophysiology and therapy of myelodysplastic syndromes}

Leukemia (2007) 21, 2237-2239; doi:10.1038/sj.leu.2404868

Myelodysplastic syndromes (MDS), whose pathophysiology remains poorly known, are myeloid disorders that may evolve toward acute leukemic transformation, and for which allogeneic bone marrow transplantation, largely through the 'graft versus leukemia' (GvL) effect, remains the only curative treatment. A peculiar and yet unexplained relationship between MDS and immune disorders including hypo- or hyper- $\gamma$-globulinemia, peripheral lymphopenia, abnormal B- or T-cell functions, monoclonal gammopathy, autoantibodies or diseases like relapsing polychondritis, vasculitis and seronegative arthritis has been noticed. ${ }^{1,2}$ The immune system also seems to contribute, in some cases, to the progressive cytopenias observed in MDS. ${ }^{3}$ On the basis of those observations, a role of the immune system in the pathophysiology and progression of MDS has been envisaged. Furthermore, innate immunity cells could offer an alternative to bone marrow transplantation in hematological malignancies including MDS, as promising results of cellular immunotherapy have been obtained in solid tumors.

Cellular components of the innate immune system, including natural killer (NK) and $\gamma \delta$ T cells have been shown to regulate cancer development and to play an important role in the lysis of transformed cells. ${ }^{4} \mathrm{NK}$ cells do not require prior immunization and are involved in the early phase of the immune response. Their activation relies on natural cytotoxicity receptors (NCR) that recognize yet unknown ligands on tumor cells. In addition, NK cells express NKG2D, an activating receptor that recognize danger signals including stress molecules (MICA/B molecules, UL-binding protein). NK also sense the level of HLA-I molecule expression through inhibitory receptors to discriminate normal from transformed cells. ${ }^{5}$ Thus, the balance between activating signals and negative signals induced by the inhibitory receptors governs the final decision to induce or not the NK-cell activation. ${ }^{6} \mathrm{NK}$ cells differentiate from a bone marrow precursor belonging to the T/NK lymphoid lineage. Several laboratories, including ours, have shown that it was possible to reproduce in vitro the differentiation of mature NK cells from CD34+ hematopoietic progenitors cultured with a combination of SCF and IL-2 and/or IL-15. ${ }^{7,8}$ In those conditions, CD34+ precursors proliferate and differentiate into cytotoxic NK cells leading to large expansion of NK cells. ${ }^{8}$

Different lines of evidence suggest that NK cells participate in the anti-leukemic immune response. Several reports have indicated an inverse relationship between NK-cell number or activity and prognosis in acute leukemia. Decreased NK-cell function along disease progression was reported in chronic myeloid leukemia $(\mathrm{CML})$ and acute myeloid leukemia (AML) patients. ${ }^{9,10}$ Experimental results outline that $\mathrm{Bcr} / \mathrm{Abl}$ leukemic targets are recognized and efficiently killed by NK cells ${ }^{11,12}$ and outline the role of NKG2D-activating receptor. ${ }^{13}$ In addition, recent clinical results support a role for alloreactive NK cells in the GvL effect observed in HLA-haploidentical transplants, and the therapeutic possibilities to manipulate NK receptor-ligand interaction to increase leukemia cell destruction are emerging. ${ }^{14}$

Non-classical T-cell receptor (TCR) $\gamma \delta \mathrm{T}$ cells represent another important component of the innate immune system. As cytotoxic effectors, $\gamma \delta$ T cells may be involved in the antitumor potential against lymphoma and multiple myeloma. ${ }^{15}$ In addition, $\gamma \delta$ T cells are regulatory cells through the secretion of pro-inflammatory cytokines ${ }^{16}$ and their potential role as antigen presenting cells. ${ }^{17}$ Peripheral $\gamma \delta$ T cells can be expanded in vivo and in vitro by a potent synthetic phosphoantigen-specific TCR $\gamma \delta$ agonist, the BrHPP molecule, ${ }^{16}$ and acquire antitumor functions. $^{18}$

The possibility to differentiate NK cells in vitro has raised the question of NK-cell differentiation in patients with myeloid disorders. We showed that the in vitro development of NK cells from CML progenitors was altered, ${ }^{19}$ suggesting that such alterations may lead to the decreased function of peripheral NK cells described in CML patients. We also found a severe alteration in NK-cell differentiation from CD34 + cells derived from MDS patients. ${ }^{20}$ None of the 14 cultures from bone marrow-purified MDS CD34+ cells resulted in NK-cell differentiation. Selection of more immature CD34+/CD38progenitors and limiting dilution experiments showed that cloning frequency of NK progenitors was at least 10 times lower than that usually found in normal bone marrow. A decreased frequency of NK progenitors in MDS bone marrow might in part account for the dramatic alteration of NK-cell differentiation in response to SCF and IL-15 in vitro.

One year ago, we published the first comprehensive study on NK cells in MDS patients, based on recent developments made in NK-cell biology. ${ }^{21}$ We studied expression of specific NKactivating and inhibitory receptors, which tightly control their cytolytic function in donor NK cells, as well as MDS-NK cell survival ex vivo, and their belonging to the myelodysplastic clone. Functional capacities of MDS NK cells were measured both in peripheral blood mononuclear cells (PBMC) and in immunoselected NK cells activated with interleukin (IL)-2. We showed that peripheral NK cells from MDS patients poorly killed K562 target cells as well as the MDS-derived cell line P39, and displayed decreased secretion of IFN $\gamma$ and TNF $\alpha$ in response to NCR triggering. Purified NK cells displayed deeply altered NK function in all MDS cases (regardless of subtype, cytogenetics or risk category), suggesting that this defect was a hallmark of MDS rather than a marker of more advanced disease.

A recent paper by Epling-Burnette et al. ${ }^{22}$ in a similar cohort of patients confirms the deeply altered NK cytolytic function we described in MDS. However, some results diverge between the two reports. For example, contrary to our study, the decreased lytic function measured in PBMC was correlated with high-risk disease and reduced NKG2D-activating receptor expression by NK cells. Although the authors used elegant correlations between percentages of NK cells in PBMC and cytotoxicity, a non-specific lysis due to other effectors could not be ascertained unless purified NK cells were used, and such assays were performed only in two patients. In our study, although more 
severe defects were observed in high-risk MDS using resting PBMC, we observed no correlation between highly decreased cytolytic function and NCR expression or disease characteristics when we assessed the functions of immunoselected NK cells. In fact, we found that NK-cell function was severely altered in spite of normal NCR expression. We recently assessed the lytic potential of NK cells by measuring their degranulation capacities (externalization of CD107a by flow cytometry assays) in response to target stimulation. ${ }^{23}$ This sensitive assay performed on PBMC but allowing specific analysis of degranulating resting NK cells confirmed our previous data. Such findings seem to be a characteristic of MDS, because in other hematological malignancies including $\mathrm{CML}$ and $\mathrm{AML}$, decreased function was associated with low level of NCR expression on NK cells. ${ }^{24}$ Finally, we showed that a significant proportion of MDS NK cells belonged to the myelodysplastic clone, as the chromosomal aberration was found in circulating NK cells from patients with either $5 q-,-7$ or +8 .

Altogether, those two studies clearly show that cytotoxicity of NK cells is highly decreased in MDS. However, the mechanisms leading to such alterations are not fully explained. The decreased expression of NKG2D, found by Epling-Burnette et al. $^{22}$ but not in our study, cannot account by itself for the peripheral NK-cell anergy in MDS. Among other possible mechanisms, we found that a significant proportion of circulating NK cells in MDS were derived from myelodysplastic progenitors (bearing the same chromosomal abnormalities) and displayed high level of apoptosis. Finally, absence of MDS-NK proliferation after stimulation with IL-2 in vitro was described in the two studies.

Those alterations of NK cells in MDS prompted us to investigate other components of the innate immune system, the $\gamma \delta$ T cells. In a large cohort of MDS patients, we recently evaluated their phenotype, function and in vitro expansion. We found that this cell population was significantly decreased in peripheral blood of the subgroup of MDS patients presenting with associated autoimmune disorders (such as rheumatoid arthritis, systemic lupus erythematosus or several autoantibodies). However, in vitro stimulation with BrHPP induced expansion of $\mathrm{V} \delta 2 \gamma \delta \mathrm{T}$ cells in $60 \%$ of MDS, including highrisk patients. Those expanded $\mathrm{V} \delta 2 \mathrm{~T}$ cells exhibited normal cytolytic function toward leukemic and MDS cell lines, and $\mathrm{FISH}$ analysis indicated that they were not derived from the MDS clone. Although we could expand the $\gamma \delta$ T cell population in MDS, their subsequent proliferation in response to BrHPP and IL-2 was significantly decreased compared to normal donors. This common proliferation defect described in NK and $\gamma \delta$ T cells in MDS occurred despite normal expression level of IL-2 receptors (unpublished data), and suggests involvement of actors of IL-2 signaling pathway downstream of the IL-2 receptor.

Our study and that of Epling-Burnette et al. ${ }^{22}$ suggest that alteration of NK-cell function may be an important factor in MDS pathophysiology and progression. Our recent results on $\gamma \delta$ $\mathrm{T}$ cells further support the hypothesis of defective immune surveillance in MDS, a concept now well established in solid tumors. ${ }^{25}$ Those observations open new perspectives not only in the understanding of MDS pathophysiology, but also in the emerging area of immunotherapy. The basic advances made in the understanding of interactions between immune system and tumors have so far yielded only limited success using vaccines or adoptive immunotherapy. MDS are malignant diseases with several characteristics suggesting that they could be good candidates for immunotherapy, including a chronic phase with low tumor burden, a clearly demonstrated GvL effect, and the efficacy of immunosuppressive therapy in some cases. A comprehensive approach to define mechanisms of resistance or escape of myelodysplastic cells to the immune system may help in the search for effective therapies as alternatives to bone marrow transplantation or conventional chemotherapy.

\section{Acknowledgements}

This work was supported by grants from INSERM, La Fondation de France (Comité Leucémie) and l'Association Laurette Fugain.

J-J Kiladjian ${ }^{1,2}$, P Fenaux ${ }^{2}$ and A Caignard ${ }^{1}$
${ }^{1}$ INSERM U753, Institut Gustave Roussy, 39 rue Camille
Desmoulins, Villejuif, France and
${ }^{2}$ AP-HP, Hôpital Avicenne, Service d'Hématologie Clinique;
Paris 13 University, Bobigny, France
E-mail: jean-jacques.kiladjian@avc.aphp.fr

\section{References}

1 Enright H, Jacob HS, Vercellotti G, Howe R, Belzer M, Miller W. Paraneoplastic autoimmune phenomena in patients with myelodysplastic syndromes: response to immunosuppressive therapy. Br J Haematol 1995; 91: 403-408.

2 Hebbar M, Kozlowski D, Wattel E, Mastrini S, Dievart M, Duclos B et al. Association between myelodysplastic syndromes and inflammatory bowel diseases. Report of seven new cases and review of the literature. Leukemia 1997; 11: 2188-2191.

3 Molldrem JJ, Caples M, Mavroudis D, Plante M, Young NS, Barrett AJ. Antithymocyte globulin for patients with myelodysplastic syndrome. Br J Haematol 1997; 99: 699-705.

4 Cerwenka A, Lanier L. Natural killer cells, viruses and cancer. Nat Rev Immunol 2001; 1: 41-49.

5 Long E. Regulation of immune responses through inhibitory receptors. Annu Rev Immunol 1999; 17: 875-904.

6 Farag S, Fehniger T, Ruggeri L, Velardi A, Caligiuri M. Natural killer cell receptors: new biology and insights into the graft-versusleukemia effect. Blood 2002; 100: 1935-1947.

7 Mrozek E, Anderson P, Caligiuri MA. Role of interleukin-15 in the development of human CD56+ natural killer cells from CD34+ hematopoietic progenitor cells. Blood 1996; 87: 2632-2640.

8 Carayol G, Robin C, Bourhis J, Bennaceur-Griscelli A, Chouaib S, Coulombel $L$ et al. NK cells differentiated from bone marrow, cord blood and peripheral blood stem cells exhibit similar phenotype and functions. Eur J Immunol 1998; 28: 1991-2000.

9 Verfaillie C, Kay N, Miller W, McGlave P. Diminished A-LAK cytotoxicity and proliferation accompany disease progression in chronic myelogenous leukemia. Blood 1990; 76: 401-408.

10 Costello RT, Sivori S, Marcenaro E, Lafage-Pochitaloff M, Mozziconacci MJ, Reviron D et al. Defective expression and function of natural killer cell-triggering receptors in patients with acute myeloid leukemia. Blood 2002; 99: 3661-3667.

11 Baron F, Turhan AG, Giron-Michel J, Azzarone B, Bentires-Alj M, Bours $\mathrm{V}$ et al. Leukemic target susceptibility to natural killer cytotoxicity: relationship with BCR-ABL expression. Blood 2002; 99: 2107-2113.

12 Cebo C, Voutsadakis IA, Da Rocha S, Bourhis JH, Jalil A, Azzarone B et al. Altered IFNgamma signaling and preserved susceptibility to activated natural killer cell-mediated lysis of BCR/ABL targets. Cancer Res 2005; 65: 2914-2920.

13 Cebo C, Da Rocha S, Wittnebel S, Turhan AG, Abdelali J, CaillatZucman $\mathrm{S}$ et al. The decreased susceptibility of $\mathrm{Bcr} / \mathrm{Abl}$ targets to NK cell-mediated lysis in response to imatinib mesylate involves modulation of NKG2D ligands, GM1 expression, and synapse formation. J Immunol 2006; 176: 864-872.

14 Ruggeri L, Aversa F, Martelli MF, Velardi A. Allogeneic hematopoietic transplantation and natural killer cell recognition of missing self. Immunol Rev 2006; 214: 202-218.

15 Wilhelm M, Kunzmann V, Eckstein S, Reimer P, Weissinger F, Ruediger $\mathrm{T}$ et al. Gammadelta $\mathrm{T}$ cells for immune therapy of patients with lymphoid malignancies. Blood 2003; 102: 200-206. 
16 Das H, Wang L, Kamath A, Bukowski J. Vgamma2Vdelta2 T-cell receptor-mediated recognition of aminobisphosphonates. Blood 2001; 98: 1616-1618.

17 Brandes $M$, Willimann $K$, Lang $A B$, Nam KH, Jin C, Brenner MB et al. Flexible migration program regulates gamma delta $\mathrm{T}$-cell involvement in humoral immunity. Blood 2003; 102: 3693-3701.

18 Viey E, Laplace C, Escudier B. Peripheral gammadelta T-lymphocytes as an innovative tool in immunotherapy for metastatic renal cell carcinoma. Expert Rev Anticancer Ther 2005; 5: 973-986.

19 Carayol G, Giron-Michel J, Azzarone B, Castagna L, Cambier N, Mishal Z et al. Altered natural killer cell differentiation in CD34+ progenitors from chronic myeloid leukemia patients. Oncogene 2000; 19: 2758-2766.

20 Kiladjian J, Bourgeois E, Bourhis J, Chouaib S, Fenaux P, Caignard A. Impaired natural killer cell differentiation in myelodysplastic syndromes. Blood 2003; 102(Suppl 1): 162a.
21 Kiladjian JJ, Bourgeois E, Lobe I, Braun T, Visentin G, Bourhis JH et al. Cytolytic function and survival of natural killer cells are severely altered in myelodysplastic syndromes. Leukemia 2006; 20: 463-470.

22 Epling-Burnette PK, Bai F, Painter JS, Rollison D, Salih HR, Krusch M et al. Reduced natural killer (NK) function associated with high-risk myelodysplastic syndrome (MDS) and reduced expression of activating NK receptors. Blood 2007; 109: 4816-4824.

23 Penack O, Gentilini C, Fischer L, Asemissen AM, Scheibenbogen C, Thiel $\mathrm{E}$ et al. CD56dimCD16neg cells are responsible for natural cytotoxicity against tumor targets. Leukemia 2005; 19: 835-840.

24 Costello RT, Fauriat C, Sivori S, Marcenaro E, Olive D. NK cells: innate immunity against hematological malignancies? Trends Immunol 2004; 25: 328-333.

25 Malmberg KJ. Effective immunotherapy against cancer: a question of overcoming immune suppression and immune escape? Cancer Immunol Immunother 2004; 53: 879-892. 